\title{
NOTE ON THE RESISTANCE OF RADIOTELEGRAPHIC ANTENNAS
}

By L. W. Austin.

According to the theory the portion of the resistance of an earthed antenna due to its radiation is ${ }^{1}$

$$
R_{\mathrm{r}}=\mathrm{I} 60 \pi^{2} \frac{h^{2}}{\lambda^{2}}
$$

which is approximately $1600 \frac{h^{2}}{\lambda^{2}}$

Where $h$ is the height from the earth to the center of capacity of the antenna and $\lambda$ the wave length, assuming that the earth below the antenna is a good conductor like salt water. As a matter of fact, in most land stations the effective height is less than the actual height and in many cases not more than one-half of it. In all antennas, however, radiation resistance must, according to the theory, decrease as the wave length increases and in accordance with the square law.

It was first noted by C. Fischer ${ }^{2}$ that in certain cases the resistance $^{3}$ of antennas increased as the period was increased by introducing inductance. Fischer believed that this indicated an increase of radiation with wave length.

The whole course of the phenomenon has been studied by the United States Naval Radio Laboratory and the results published. ${ }^{4}$

Fig. I shows the resistance curve of the antenna used by the naval laboratory at the Bureau of Standards and that of the U.S. S. Maine. The first is typical of a land station with poor ground conditions, the curve falling rapidly as the true radiation resistance drops with increasing wave length and then rising

${ }^{1}$ Zenneck Lehrbuch d. drahtlosen Telegraphie, I913, p. 202.

2 Phys. Zeit. I9II, p. 295.

${ }^{3}$ Leaving out of account the resistance of the inductance coils.

4 Proc. Wash. Acad. I, p. 9, I91r; and this Bulletin, 9, p. 65, 1912; Scientific Paper No. I89. 
again in a straight line as the wave length is further increased. The curve for the Maine shows the same drop at the shorter wave lengths followed by a rise which is very slight. Fig. 2 shows the antenna resistance of the high power station at Arlington, Va., with the towers grounded. Here there is almost no rise as the wave length is increased, showing how perfect ground conditions can be made by the use of an extensive network of ground wires.

$U p$ to the present there has been no satisfactory explanation of the rise of the antenna resistance curve, although it seems certain that it has something to do with the ground. Experiments by $\mathrm{H}$. True ${ }^{5}$ show, however, that the ground resistance, as indicated by fall of potential methods, is greater at short than at long wave lengths. If, however, we consider the ground as a dielectric rather than as a conductor and consider it as a portion of the total dielectric lying between the antenna, regarded as the upper plate of a condenser, and the ground water, regarded as the lower plate, we reach a very probable explanation of the peculiar form of many antenna resistance curves. For it is well known that the equivalent resistance of an imperfect dielectric increases as the wave length is increased.

As an example ${ }^{6}$ of experiments showing this, the equivalent resistance of a certain glass condenser of approximately $0.002 \mathrm{mf}$ capacity measured in the Naval Radio Laboratory is shown in Fig. 3. The equivalent resistance of the condenser at the various wave lengths is determined by placing it in a circuit acted upon by a buzzer-excited wave meter. The current in the circuit containing the condenser is measured by means of a thermoelement. The condenser under test is then replaced by a variable air condenser adjusted to the same capacity in series with which resistance is introduced until the thermoelement indicates the same high frequency current as in the case of the glass condenser. The series resistance then represents the equivalent resistance of the glass condenser. Fig. 3 shows that the equivalent resistance of the glass condenser increases in direct proportion to the wave length, just as does the resistance of the antenna at the Bureau of Standards beyond I 500 meters.

${ }^{5} \mathrm{Jahrbuch}$ d. drahtlosen Telegraphie, V, p. I25, I9Ir.

- Proc. of the Inst. of Radio Engineer, I, p. 35, r913. 


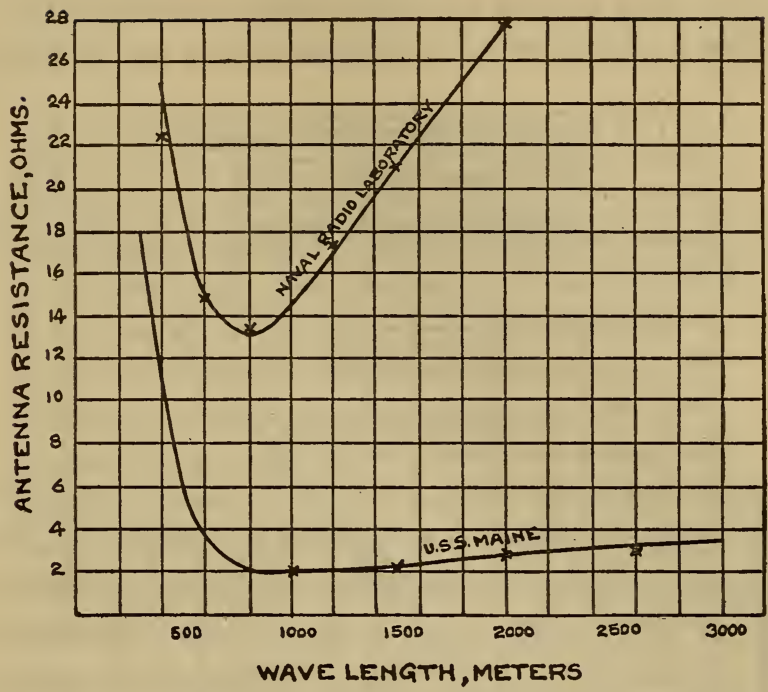

FIg. I.-Antenna resistance, Naval Radio Laboratory, U.S. S. "Maine"

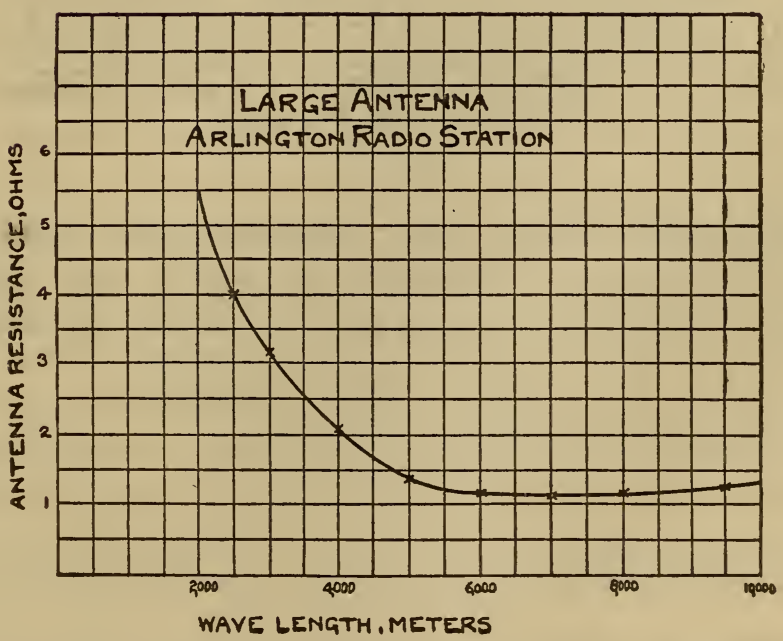

FIG. 2 
The rise in the resistance curve of the Maine, as shown in Fig. $I$, is probably due to the fact that the measurements were made with the ship in dock, as it is impossible to use sufficiently sensi-

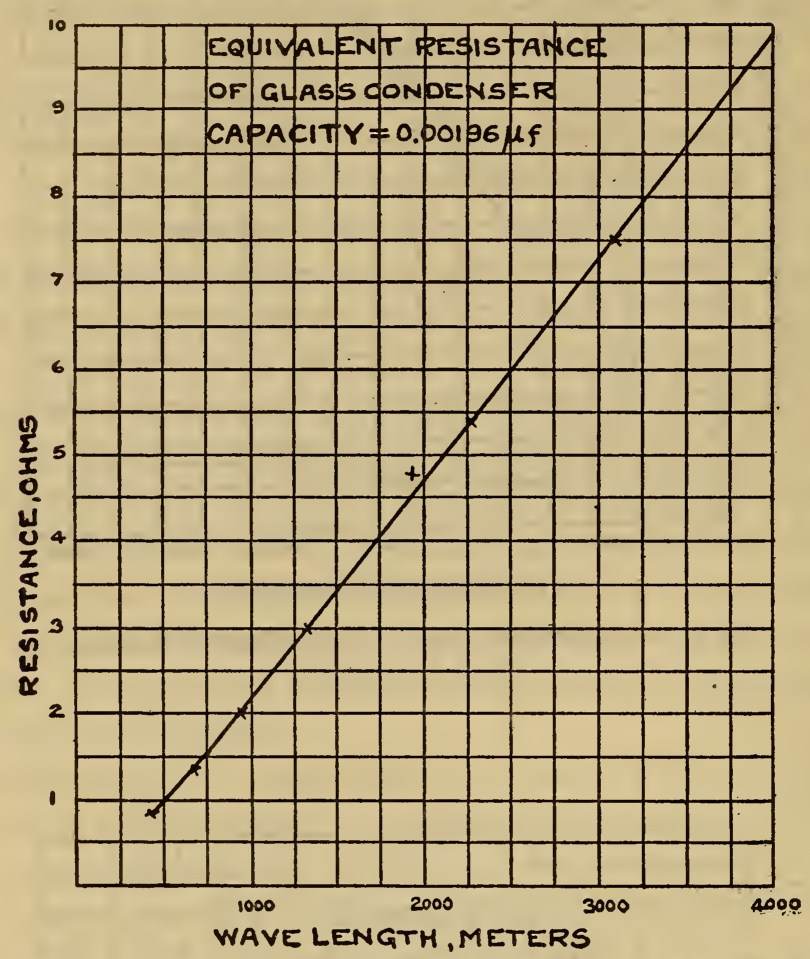

Fig. 3 .

tive galvanometers under other circumstances. With the ship in dock, of course, a considerable portion of the field passes through poor dielectric material before reaching the water.

U. S. NAVAL RADIOTELEGRAPHIC LABORATORY, WASHINGTON, February I3, I9I 5 . 